\title{
Mineração de dados no fluxo de processos que impactam na arrecadação tributária em Curitiba
}

\section{Data mining on flow of processes that impact tax collection in Curitiba}

\author{
Adolfo José Macarini Filho \\ Prefeitura Municipal de Curitiba - Secretaria do Governo Municipal - SGM - Curitiba - Brasil \\ amacarini@sgm.curitiba.pr.gov.br \\ Andrea Cristina Lima Duarte Ferreira \\ Prefeitura Municipal de Curitiba - Secretaria do Governo Municipal - SGM - Curitiba - Brasil \\ aduarte@imap.curitiba.pr.gov.br \\ Elziane Cazura Xavier \\ Prefeitura Municipal de Curitiba - Secretaria do Governo Municipal - SGM - Curitiba - Brasil \\ ecazura@sit.curitiba.pr.gov.br \\ Deborah Ribeiro Carvalho \\ Pontifícia Universidade Católica do Paraná - PUCPR - Curitiba - Brasil \\ drdrcarvalho@gmail.com
}

\section{Resumo}

Este artigo objetiva identificar os potenciais gargalos que possam retardar a tramitação de processos vinculados à arrecadação tributária de Curitiba. Para tal foi adotado o conjunto dos trâmites de processos entre 2013 e 2015, do Sistema Único de Protocolos. A partir dos dados sistematizados é possível identificar a unidade de origem e a de destino de cada tramitação, bem como o tempo demandado entre ambas. Sobre este conjunto de dados foram descobertas regras de associação, que constitui uma das tarefas de mineração de dados. A partir das regras descobertas foi possível identificar um alto índice de recursividade. Esta recursividade na tramitação pode indicar um gargalo importante, contribuindo para o retardo na tramitação dos processos. As associações descobertas, envolvendo origem e destino das tramitações, permitem identificar o caminho percorrido por um processo possibilitando orientar os pontos críticos a serem revisados e aperfeiçoados nos fluxos. Foram identificadas como maiores gargalos, unidades, pelas quais tramitam processos de Revisão de Valores de ITBI, Unificação/Subdivisão, Expedição de Alvará de Construção, Fiscalização COSEDI e Certidão de IPTU, cujos tempos de permanência entre algumas unidades administrativas variaram de zero a 1.152 dias. Este tempo de permanência demonstra a inexistência ou mesmo o desconhecimento de processos de trabalho mapeados e de normativas específicas. Esta pesquisa motiva novos estudos em outros segmentos da administração pública, inclusive com identificação de não apenas de gargalos, mas também a respectiva janela temporal de permanência entre as unidades administrativas.

Palavras-chave: Descoberta de regras de associação, fluxo de processos, arrecadação tributária. 


\section{Abstract}

This paper aims to identify possible problems that may delay the city of Curitiba tax collection processing. To this end, the procedures set between 2013 and 2015, of the Single System of Protocols, are adopted. From the systematized data, it is possible to identify the unit of origin and destination of each processing, as well as the time required between both. In this data set were discovered association rules, which is one of the data mining tasks. Of the rules discovered and possible information in a high index of recursion. This recursion in the process can indicate a significant bottleneck, contributing to the delay in the processing of the processes. As discovered associations, involving the origin and destination of the dolls, we identify the path traveled by a process that allows us to guide the critical points to be reviewed and improved in the flows. As the associations discovered, involving the origin and destination of the dolls, we identified the path followed by a process that allows us to guide the critical points to be reviewed and improved in the flows. The main bottlenecks, identified by ITBI, Unification / Subdivision, Construction Permit Expedition, COSEDI Inspection and IPTU Certificate, were defined as main problems, and the length of stay between some administrative units ranged from zero to 1,152 days. This time of permanence in some units, demonstrates an inexistence or ignorance of specific work processes and specifications. This research motivates new studies in other segments of the public administration, including not only bottlenecks, but also the temporary windows of permanence between administrative units.

Keywords: Discovery of association rules, flow of processes, tax collection data mining.

\section{Introdução}

A arrecadação tributária municipal tem apresentado índices mais baixos a cada ano (CRUZ, 2015), impactando o cumprimento do repasse por parte das esferas de governo federal e estadual (AUDI, 2014). O município de Curitiba também vem apresentando queda na arrecadação (Gráfico 1).

Considerado o período entre 2013 a 2015, constatou-se que 59,6\% do volume de processos tramitados, no município de Curitiba, envolvendo questões que podem estar atreladas à arrecadação tributária, demandaram entre zero e 100 dias. Vale destacar que a média de tempo de tramitação é de 162 dias. Avaliando a média para cada ano individualmente, observa-se uma redução de 56,7\% de 2013 para 2015, em função da constante preocupação em aperfeiçoar a tramitação de processos (IMAP, 2013).

Gráfico 1: Receita estimada e arrecadação efetiva no Município de Curitiba 2010 - 2015

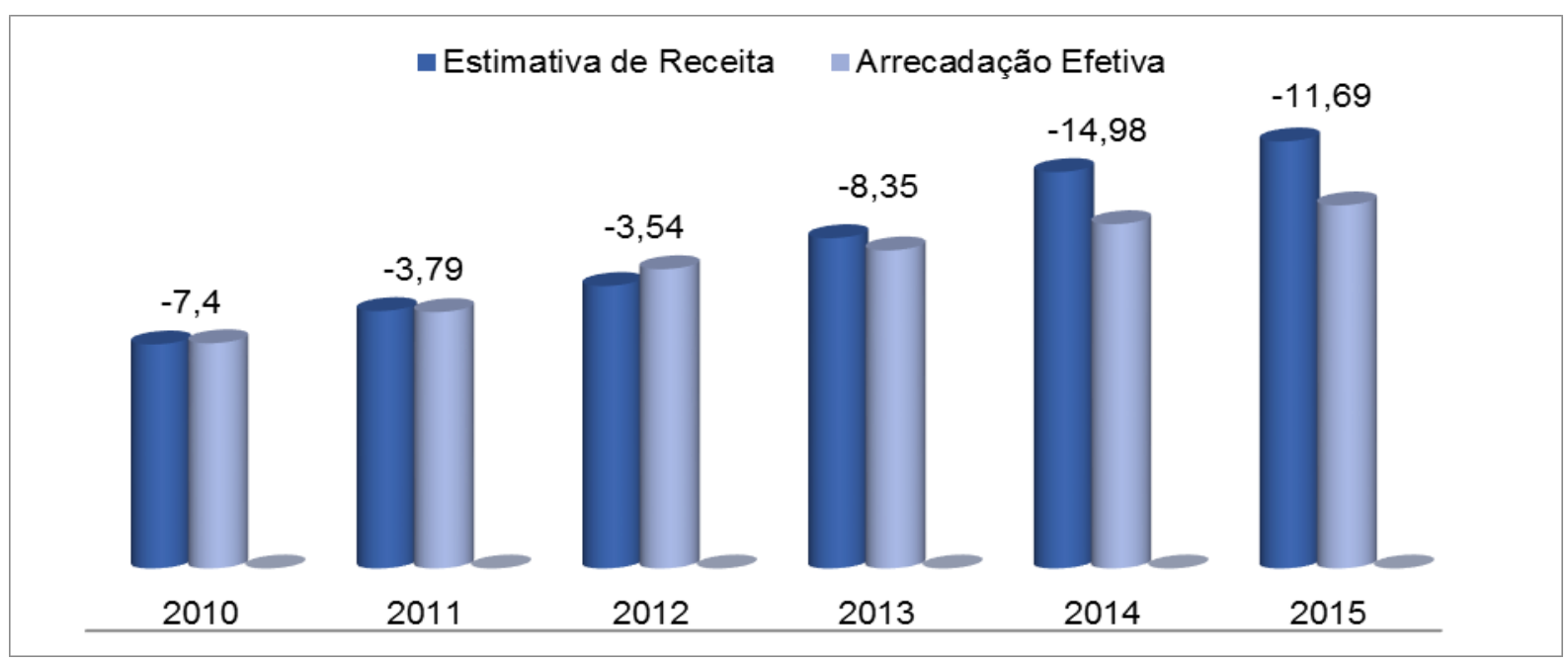

Fonte: Portal da Transparência (2016) 
Dada à ausência de fluxos eletronicamente estabelecidos, estão sendo pesquisados por fatores contribuam para com a morosidade na tramitação de processos. Porém, a tramitação de processos representa um conjunto de dados de grande magnitude e complexidade. Por exemplo, entre 2013 e 2015, tramitaram cerca de 270 mil processos, gerando em torno de dois milhões de registros, tendo como o tempo médio entre a abertura e encerramento, 162 dias. Ou seja, não constitui uma tarefa trivial identificar os pontos de gargalos que contribuem para uma possível morosidade.

Aliado ao fato que a evolução da Tecnologia da Informação e Comunicação - TIC contribuiu para a coleta e armazenamento de grandes volumes de dados. A grande quantidade de dados e a complexidade de seus respectivos relacionamentos dificultam a identificação de padrões e comportamentos, por parte dos administradores públicos e responsáveis pela tomada de decisão (MARQUES, 2011).

Já existem experiências que mostram que as técnicas de mineração de dados possibilitam aperfeiçoar os processos e dar agilidade à tramitação de documentos e protocolos administrativos, das instituições públicas (KUM; DUNCAN; STEWART apud GAGO JÚNIOR, 2012).

Esta pesquisa propõe a descoberta de potenciais gargalos na tramitação de processos que impactam na gestão tributária municipal, a partir da mineração de dados. Dado o volume e complexidade dos dados envolvidos, as estratégias tradicionais de extração e informação das bases de dados podem não ser suficientes. Sendo assim, existem estratégias alternativas baseadas na Inteligência Artificial, tais como, o processo de KDD (Knowledge Discovery in Databases) que constitui um conjunto de etapas contínuas que objetivam extrair padrões a partir de bases de dados (FAYYAD; PIATETSKYSHAPIRO; SMYTH, 1996). Esse conjunto é composto de três etapas: pré-processamento, mineração de dados e pós-processamento.

$\mathrm{Na}$ etapa de pré-processamento, a fase de seleção de dados é a primeira no processo de descobrimento de informação e possui impacto significativo sobre a qualidade do resultado final, uma vez que nesta fase é escolhido o conjunto de dados contendo todas as possíveis variáveis (também chamadas de características ou atributos) e registros (também chamados de casos ou observações) que farão parte da análise. Normalmente essa escolha dos dados fica a critério de um especialista do domínio, ou seja, alguém que realmente entende do assunto em questão.

Esta etapa ainda compreende a fase de limpeza dos dados que é uma parte crucial no processo de KDD, pois a qualidade dos dados vai determinar a eficiência dos algoritmos de mineração. Nesta etapa deverão ser realizadas tarefas que eliminem dados redundantes e inconsistentes, recuperem dados incompletos e avaliem possíveis dados discrepantes ao conjunto (PRASS, 2004).

$\mathrm{Na}$ etapa de Mineração de Dados, podem ser identificados programas computacionais correspondentes a três tarefas principais: classificação, descoberta de regras de associação e agrupamento. O pós-processamento contempla a depuração e/ou a síntese das relações descobertas, que usualmente são denominadas de "padrões descobertos" (CARVALHO et al., 2012).

Este estudo contribui para um repensar em estratégias para melhoria e modernização dos processos de trabalho que envolve a arrecadação tributária municipal, no que se refere a uma maior agilidade nos processos. Fato que vem ao encontro com a melhoria da governança pública.

Desta forma, este estudo objetiva a descoberta de potenciais gargalos na tramitação de processos que impactam na gestão tributária municipal, a partir da mineração de dados, utilizando a base de dados do Sistema Único de Protocolos - SUP. 


\section{Encaminhamento Metodológico}

Trata-se de uma pesquisa exploratória, pois visa a descoberta, o achado, a elucidação de fenômenos ou a explicação daqueles que não eram aceitos apesar de evidentes (GONÇALVES, 2014).

O conjunto de dados trabalhado se refere aos anos de 2013 a 2015, incluindo os dois primeiros meses de 2016. Estes dados são oriundos do módulo Sistema Único de Protocolo - SUP, do Sistema de Gestão Tributária Municipal - GTM, que armazena todas as etapas pelas quais um processo tramita nas unidades administrativas da Prefeitura Municipal de Curitiba - PMC. Foram selecionados processos concluídos ou em tramitação.

$\mathrm{Na}$ etapa de pré-processamento foram selecionados os registros e as variáveis, das tramitações, a partir dos seguintes critérios:

- Estar relacionado à arrecadação tributária; e

- Apresentar mais de etapas na tramitação.

Entre os atributos selecionados (Tabela 1) destacam-se:

- Origem: de qual unidade saiu o processo.

- Destino: para qual unidade administrativa foi tramitado o processo.

- Assunto: assunto que motiva o processo.

- Data de Cadastro: data de entrada do processo, que permite calcular o tempo de tramitação.

- № do Protocolo: identifica o processo.

- Data de Tramitação: data da movimentação do processo.

- Interessado: identificar o contribuinte que deu entrada ao processo (pessoa física ou jurídica) ou o servidor público.

- Unidade de Origem: para identificar o órgão em que foi dado início o processo, independente da unidade em qual este esteja tramitando.

- Status: para identificar qual a situação em que se encontra o processo (em andamento ou encerrado), fazendo uma correlação com o tempo de permanência numa determinada unidade administrativa.

- Sequência: para identificar qual a sequência dos trâmites dos processos.

- Aceite: para compor a análise do tempo de permanência na unidade administrativa.

Por uma questão ética, a não identificação das Secretarias e respectivas unidades foram adotados códigos artificiais.

Tabela 1: Exemplo de dados disponíveis no cadastro de trâmites

\begin{tabular}{|c|c|c|c|c|c|c|c|c|c|c|}
\hline ORIGEM & DEstINO & Assunto & $\begin{array}{c}\text { DATA } \\
\text { CRIACAO }\end{array}$ & PROTOCOLO & INTERESSADO & $\begin{array}{l}\text { UNID } \\
\text { ORIGEM }\end{array}$ & $\begin{array}{l}\text { TEMP } \\
\text { VIDA }\end{array}$ & STATUS & SEQ & ACEITE \\
\hline$F-1$ & $F-12$ & $\begin{array}{c}\text { Revisão } \\
\text { Valores de } \\
\text { ITBI }\end{array}$ & $29 / 08 / 2014$ & $1-95529 / 2014$ & & $\mathrm{~F}$ & 40 & Enc & 1 & sim \\
\hline$F-12$ & $F-12$ & $\begin{array}{c}\text { Revisão } \\
\text { Valores de } \\
\text { ITBI }\end{array}$ & $29 / 08 / 2014$ & $1-95529 / 2014$ & & $\mathrm{~F}$ & 40 & Enc & 2 & Sim \\
\hline$F-32$ & $\mathrm{~F}-1$ & $\begin{array}{l}\text { Certidão de } \\
\text { IPTU via } \\
\text { Processo }\end{array}$ & $05 / 02 / 2013$ & $1-12134 / 2013$ & & $\mathrm{~F}$ & 112 & Enc & 1 & Sim \\
\hline $\mathrm{F}-1$ & $F-51$ & $\begin{array}{l}\text { Certidão de } \\
\text { IPTU via } \\
\text { Processo }\end{array}$ & $05 / 02 / 2013$ & $1-12134 / 2013$ & & $\mathrm{~F}$ & 112 & Enc & 2 & Sim \\
\hline U-B11 & U-85 & $\begin{array}{l}\text { Unificação/ } \\
\text { Subdivisão }\end{array}$ & $17 / 06 / 2013$ & $91-338 / 2013$ & & $u$ & 998 & And & 1 & $\operatorname{sim}$ \\
\hline U-85 & U-53 & $\begin{array}{l}\text { Unificação/ } \\
\text { Subdivisão }\end{array}$ & $17 / 06 / 2013$ & $1-12134 / 2013$ & & $u$ & 998 & And & 2 & $\operatorname{sim}$ \\
\hline
\end{tabular}

Fonte: Autoria própria (2017) 
Para a identificação dos possíveis gargalos foi adotada para a etapa de Mineração de Dados, a tarefa de descoberta de regras de associação. Esta opção se justifica, pois permite identificar possíveis recorrências entre a origem e destino da tramitação dos processos.

Para esta descoberta de regras de associação foi adotado o algoritmo APRIORI (BORGELT; KRUSE, 2002). As regras de associação descobertas apresentam o $\mathrm{A} \rightarrow \mathrm{C}$ $(\mathrm{X} \%, \mathrm{Y} \%)$. O que representa a associação entre os itens contidos no antecedente $(A)$ e no consequente $(C)$ da regra, lendo-se se $(A)$ então $(C)$. O X\% representa a probabilidade de $\mathrm{A}$ ocorrer. $\mathrm{Y} \%$ representa a probabilidade condicional do $\mathrm{C}$ ocorrer dado que o $\mathrm{A}$ ocorreu. As medidas $\mathrm{X} \%$ e Y\% são denominadas como Suporte e Confiança da regra, respectivamente.

Para a descoberta de regras de associação foram consideradas apenas duas variáveis, unidade de origem e de destino de tramitação do processo, bem como parametrizados os valores de suporte e confiança respectivamente 0,1 e 10. A aplicação de suporte e confiança tão baixos se deve ao fato da pesquisa estar relacionada à descoberta de padrões ou eventos não convencionais.

\section{Resultados}

O SUP contava em 2016 com uma base de 27,7 milhões de registros de trâmites de movimentações, representando 6,2 milhões de protocolos, cerca de 4 mil assuntos, sendo destes 1.853 oficiais e com 3.989 unidades administrativas de trâmites ativas, considerando os últimos 35 anos.

Durante a avaliação dos dados algumas inconsistências foram identificadas, tais como: não informação sobre o interessado, registros com tempo de vida negativo, não informação do assunto, etc. A simples identificação de situações de não conformidade dos dados apontam por situações que podem ser aperfeiçoadas no sistema (BRACHMAN; ANAND, 1996).

Foram selecionados cinco assuntos vinculados à arrecadação tributária:

Certidão de IPTU - Imposto Predial e Territorial Urbano via Processo;

Expedição de Alvará de Construção/ Alvará;

Fiscalização COSEDI - Comissão e Segurança da Edificação;

Revisão de Valores de ITBI - Imposto de Transmissão de Bens Inter vivos;

Unificação/ Subdivisão.

Os critérios de seleção dos assuntos foram determinados pela relevância da ação pública sobre o trâmite, no que tange os processos de trabalho.

Inicialmente são apresentadas três regras ( $R 1, R 2$ e $R 3$ ) relacionadas ao assunto Revisão de Valores de ITBI, considerando 1.455 processos tramitados, totalizando 14.825 trâmites:

$$
\text { R1 - ORIGEM_F-1 } \rightarrow \text { DESTINO_F-1 (22,1\%, 36,5\%). }
$$

A partir da regra R1 é possível identificar que $22,1 \%$ dos trâmites analisados, teve como origem a unidade administrativa F-1, sendo que destes, $36,5 \%$ destinou a própria F1. Fato este que ocorre em várias sequencia de trâmites e que evidencia a recursividade na tramitação deste assunto.

Foi identificada também que, a variação do tempo de permanência na unidade F-1, variou entre zero e 135 dias.

Do total de processos tramitados para este assunto, identificou-se que 57 dos 1.455, não apresentaram trâmite para a unidade $\mathrm{F}-1$, quando o interessado pelo assunto aparece como o próprio município de Curitiba. 


$$
\text { R2 - ORIGEM_F-32 } \rightarrow \text { DESTINO_F-32 (19,7\%, 42,8\%). }
$$

A partir da regra R2 é possível identificar que $19,7 \%$ dos trâmites analisados, teve como origem a unidade administrativa F-32, sendo que destes, $42,8 \%$ destinou a própria F-32. Fato este que ocorre em várias sequencias de trâmites e que evidencia a recursividade na tramitação deste assunto.

A variação do tempo de permanência nesta unidade ficou entre zero e 245 dias. Do total de processos tramitados para este assunto, identificou-se que 276 dos 1.455 , não apresentaram trâmite para a unidade F-32, demonstrando uma variação nos encaminhamentos dados aos processos.

$$
\text { R3 - ORIGEM_F-1 } \rightarrow \text { DESTINO_F-7 (22,1\%, 40,6\%). }
$$

A partir da regra R3 é possível identificar que $22,1 \%$ dos trâmites analisados, teve como origem a unidade administrativa $\mathrm{F}-1$, sendo que destes, $40,6 \%$ destinou a unidade administrativa F-7. Fato este que evidencia um fluxo normal de tramitação, onde não se percebe recursividade.

No entanto foi identificada uma variação do tempo de permanência para este trâmite entre zero e 68 dias.

Do total de 1.455 processos tramitados para este assunto, identificou-se que 480 destes não seguem este fluxo de trâmite, demonstrando uma variação nos encaminhamentos dados aos processos.

$\mathrm{Na}$ sequência apresentam-se duas regras (R4 e $\mathrm{R5}$ ) para 0 assunto Unificação/Subdivisão, considerando 1.324 processos tramitados, totalizando 10.024 trâmites.

$$
\text { R4 - ORIGEM_E-TPF } \rightarrow \text { DESTINO_E-TPF (8,1\%, 93\%). }
$$

A partir da regra R4 é possível identificar que $8,1 \%$ dos trâmites analisados, teve como origem a unidade administrativa E-TPF, sendo que destes, $93 \%$ destinou a própria E-TPF.

Foi identificada também que, a variação do tempo de permanência nesta unidade, variou entre zero e 1.144 dias.

Contudo, dos 671 processos que tiveram esta unidade administrativa como origem e destino, $100 \%$ encontram-se também em andamento.

$$
\text { R5 - ORIGEM_S-1 } \rightarrow \text { DESTINO_E-TPF }(7,9 \%, 98,1 \%) \text {. }
$$

A partir da regra R5 é possível identificar que $7,9 \%$ dos trâmites analisados, teve como origem a unidade administrativa $\mathrm{S}-1$, sendo que destes, $98,1 \%$ destinou a unidade administrativa E-TPF. Fato este que evidencia um fluxo normal de tramitação.

Constam 716 processos que apresentaram trâmite da unidade $\mathrm{S}-1$, para a unidade E-TPF, demonstrando que $54,1 \%$ do total de processos tramitados para este assunto, seguem este fluxo de trâmite.

No entanto foi identificada uma variação do tempo de permanência para este trâmite entre zero e 1.152 dias.

Observa-se que $8,9 \%$ destes processos não tiveram aceite até a data de aplicação do experimento. Por outro lado, $91,1 \%$ registraram aceite pela unidade administrativa de destino e permanecem em andamento. 
Do total de 1.324 processos tramitados para este assunto, identificou-se que 608 destes não seguem este fluxo de trâmite, demonstrando uma variação nos encaminhamentos dados aos processos.

Para o assunto Expedição de Alvará de Construção/Alvará, foram apresentadas duas regras (R6 e R7), considerando 12.846 processos e 85.452 trâmites.

$$
\text { R6 - ORIGEM_U-B } \rightarrow \text { DESTINO_U-B (7,6\%, 51\%). }
$$

A partir da regra $\mathrm{R} 6$ é possível identificar que $7,6 \%$ dos trâmites analisados, teve como origem a unidade administrativa U-B, sendo que destes, $51 \%$ destinou a própria UB. Fato este que evidencia a de recursividade na tramitação. A variação do tempo de permanência nesta unidade ficou entre zero e 1.108 dias.

$$
\text { R7 - ORIGEM_U-B11 } \rightarrow \text { DESTINO_O-RQ (6\%, 16\%). }
$$

A partir da regra R7 é possível identificar que $6 \%$ dos trâmites analisados, teve como origem a unidade administrativa U-B11, sendo que destes, $16 \%$ destinou a unidade $\mathrm{O}-\mathrm{RQ}$.

Constam 811 processos que apresentaram trâmite da unidade U-B11, para a unidade $\mathrm{O}-\mathrm{RQ}$, demonstrando que $6,3 \%$ do total de processos tramitados para este assunto, seguem este fluxo de trâmite.

No entanto foi identificada uma variação do tempo de permanência para este trâmite, que ficou entre zero e 939 dias.

Em análise aos processos do assunto Expedição de Alvará de Construção/Alvará, foi identificado que no trâmite para a unidade $O-R Q$, ocorre a entrega de documento ao interessado, evidenciando que todos os tramitem posteriores compreendem exclusivamente fluxos administrativos de digitalização e arquivamento. Estes fluxos comprometem de $85 \%$ a $90 \%$ do tempo de vida total destes processos.

Na sequência apresenta-se uma regra (R8) para o assunto Fiscalização COSEDI, considerando 735 processos tramitados, totalizando 3.714 trâmites:

R8 - ORIGEM_U-FI6 $\rightarrow$ DESTINO_U-FI6 (4\%, 89,3\%).

A partir da regra R8 é possível identificar que $4 \%$ dos trâmites analisados, teve como origem a unidade administrativa U-FI6, sendo que destes, $89,3 \%$ destinou a própria U-F16. Fato este que evidencia a recursividade na tramitação.

A variação do tempo de permanência nesta unidade ficou entre zero e 200 dias. Destaca-se que $92,3 \%$ destes processos ainda se encontram com status em andamento.

Para o assunto Certidão de IPTU via Processo, apresentam-se duas regras (R9 e R10), considerando 634 processos tramitados, totalizando 2.167 trâmites:

$$
\text { R9 - ORIGEM_F-32 } \rightarrow \text { DESTINO_F-32 (52,2\%, 21,9\%). }
$$

A partir da regra R9 é possível identificar que $52,2 \%$ dos trâmites analisados, teve como origem a unidade administrativa F-32, sendo que destes, $21,9 \%$ destinou a própria F-32. Fato este que evidencia a recursividade na tramitação.

A variação do tempo de permanência nesta unidade ficou entre zero e 574 dias. Do total de processos tramitados para este assunto, identificou-se que 84 dos 634, não apresentaram trâmite para a unidade F-32, demonstrando uma variação nos encaminhamentos dados aos processos.

$$
\text { R10 - ORIGEM_F-32 } \rightarrow \text { DESTINO_F-10 (52,2\%, 45,1\%). }
$$


A partir da regra R10 é possível identificar que $52,2 \%$ dos trâmites analisados, teve como origem a unidade administrativa F-32, sendo que destes, $45,1 \%$ destinou a unidade administrativa F-10. Fato este que evidencia um fluxo normal de tramitação, onde não se percebe recursividade.

No entanto foi identificada uma variação do tempo de permanência para este trâmite, que ficou entre zero e 48 dias.

\section{Discussão}

A partir dos resultados observa-se uma discrepância nos fluxos de tramitação entre as unidades administrativas, apresentando alto índice de recursividade, recorrência em todos os assuntos analisados, e variação do seu tempo de vida, conforme as regras R1, R2, R4, R6, R8 e R9.

O excessivo tempo de vida, identificado em alguns processos, pode evidenciar ruído nos dados, como por exemplo, a falta de registro de baixa no Sistema, por parte da unidade administrativa responsável pelo trâmite, após o arquivamento do documento físico, hipótese confirmada na etapa de mineração pelas regras R4 e R7.

Processos tramitados no mesmo dia ou com trâmite único, não são indicativos de eficiência e efetividade na tramitação, uma vez que existem processos que se encerram em menos de um dia com "n" trâmites e outros que num único trâmite são encerrados, porém o tempo de permanência na unidade administrativa é demasiadamente elevado.

Evidencia-se que uma mesma unidade administrativa que apresenta recursividade num determinado assunto, pode também apresentar recursividade de forma recorrente em outros processos de outros assuntos.

Um fato que determina a recursividade para uma mesma unidade administrativa pode estar associado à imposição do sistema, para a anexação de informações e documentos. No entanto, conforme o Decreto Municipal o 1.111/2004 (CURITIBA, 2004) não reconhece este procedimento como uma prática regulamentada. Vale destacar que este Decreto dispõe sobre a unificação dos procedimentos nos Protocolos dos Órgãos Municipais e delibera em seus artigos 15, 16 e 17 sobre o tempo de permanência dos processos nas unidades administrativas.

Outra situação que contribui para o elevado tempo de vida de alguns processos, é justificada pela necessidade de aguardar a entrega de documentação ou quitação de débito, dependentes do contribuinte, (regras R1, R2 e R4).

A inexistência ou desconhecimento de processos de trabalho mapeados e de normativas específicas contribuem para a diversidade em relação ao tempo de tramitação, para os diversos assuntos e processos (regras R3, R5, R9 e R10).

Ressalta-se a falta de encerramento dos processos do assunto Unificação/Subdivisão, uma vez que $94,9 \%$ do total de processos tramitados encontramse ainda em andamento (regra R4).

\section{Conclusão}

O presente estudo conclui que os possíveis gargalos que podem impactar no processo de arrecadação tributária municipal são: a recursividade nos trâmites dos processos, a variação nos fluxos dos trâmites, a variação do tempo de permanência nas unidades administrativas, bem como inconsistências da base de dados do SUP.

Exemplificando, os demonstrados nos tramites de processos de Revisão de Valores de ITBI, Unificação/Subdivisão, Expedição de Alvará de Construção, Fiscalização COSEDI e Certidão de IPTU. 
A participação de um especialista de domínio auxiliando no processo de seleção dos dados foi fundamental para o encaminhamento do estudo (PRASS, 2004), dada a expressiva quantidade de assuntos, principalmente no que diz respeito à identificação daqueles que poderiam estar vinculados ao tema arrecadação tributária. Entretanto, não dispor de uma equipe de especialistas de outras prefeituras, inviabiliza a realização de um estudo comparativo.

Destacam-se alguns pontos que dificultaram o processo de realização da pesquisa, como: a falta de atributo que vinculasse os assuntos à arrecadação tributária, a inconsistência na base de dados, a manipulação em relação ao grande volume de dados, a falta de referenciais teóricos relacionados ao tema proposto no estudo e o fato de não ser considerada a janela temporal de permanência dos processos nas unidades administrativas.

Conclui-se então que este estudo contribui para a melhoria dos processos de trabalho, fundamentando os pontos base para a revisão de fluxos e trâmites, sugerindo e apoiando a implantação de uma solução de BPM - Bussiness Process Management, processo de negócio que coordena o comportamento das pessoas, sistemas, informações e coisas para produzir resultados em apoio à estratégia de negócios (GARTNER, 2016).

Além disto, esta pesquisa fornece subsídios para que sejam realizados outros estudos com um universo de pesquisa mais amplo, considerando outras variáveis e aplicando técnicas que analisem a temporalidade, a partir de estratégias de Mineração de Processos. Por exemplo, pesquisar o comportamento do usuário em relação ao uso do SUP; aplicação de técnicas de mineração, com regras de temporalidade para descoberta de padrões que não foram contemplados neste estudo; análise comparativa entre os resultados dos estudos sugeridos e realizados, bem como, estudos voltados a outros segmentos ou áreas da administração pública municipal.

\section{Referências}

BORGELT, C.; KRUSE, R. Induction of association rules: apriori implementation. In: HARDLE, W.; RONZ, B. (Org.) Compstat. Heidelberg: Physica-Verlag, 2002.

BRACHMAN, R. J.; ANAND, T. The process of knowledge discovery in databases: A First Sketch. AAAl-94 Workshop on Knowledge Discovery in Databases, 1996. Disponível em: <http://aaai.org/Papers/Workshops/1994/WS-94-03/WS94-03-001.pdf>. Acesso em: 04 abr. 2016.

CARVALHO, D. R. et al. Mineração de dados aplicadas à fisioterapia. Fisioterapia do Movimento, Curitiba, v. 25, n. 3, p. 595-605, jul. /set. 2012.

CURITIBA. Decreto Municipal no 1.111 de 30 de novembro de 2004. Confere o artigo 72, inciso IV e V da lei orgânica do município de Curitiba. Palácio de 29 de março, Curitiba, $30 \quad$ nov. 2004.2 Disponível em: <http://multimidia.curitiba.pr.gov.br/2010/00082698.pdf>. Acesso em: 04 abr. 2016.

IMAP - Instituto Municipal de Administração Pública. Proposta - Manual de Volumetria PMC 2013 (Documento de Circulação Restrita). Curitiba: IMAP, 2013..

CRUZ, D. Mesmo com aumento de tributos, arrecadação tem queda de $2,44 \%$. G1, jul. 2015. Economia. Disponível em: <http://g1.globo.com/economia/noticia/2015/07/mesmocom-aumento-de-tributos-arrecadacao-tem-queda-de-244.html>. Acesso em: 05 set. 2016. 
FAYYAD, U; PIATETSKY-SHAPIRO, G; SMYTH, P. From Data Mining to Knowledge Discovery in Databases. Al Magazine, v. 17, n. 3, p. 37-54, 1996. Disponível em: <http://www.csd.uwo.ca/faculty/ling/cs435/fayyad.pdf>. Acesso em: 12 abr. 2016.

AUDI, A. Curitiba arrecada menos e prevê aperto de cintos. Gazeta do Povo, set. 2014. Vida Pública. Disponível em: <http://www.gazetadopovo.com.br/vida-publica/curitibaarrecada-menos-e-preve-aperto-de-cintos-eeb159txacml80p4i711zye6m>. Acesso em: 16 fev. 2016.

GAGO JUNIOR, E. L. A. Mapas auto-organizáveis aplicados em governo eletrônico. 2012. 80 f. Dissertação (Mestrado em Engenharia Elétrica) - Faculdade de Engenharia Elétrica e de Computação, Universidade Estadual de Campinas, São Paulo.

GARTNER. IT Glossary. 2016. Disponível em: <http://www.gartner.com/itglossary/business-process-management-bpm>. Acesso em: 19 abr. 2016.

GONÇALVES, H. A. Manual de metodologia da pesquisa científica. 2. ed. São Paulo: Avercamp, 2014.

MARQUES, E. Z. Uma proposta de utilização das tecnologias de business intelligence para suporte a tomada de decisão no contexto de governo eletrônico. 2011. 348 f. Dissertação (Mestrado em Engenharia Elétrica) - Faculdade de Engenharia Elétrica e de Computação, Universidade Estadual de Campinas, São Paulo.

PORTAL DA TRANSPARÊNCIA. Acesse e Acompanha as contas públicas. Disponível em: <http://www.transparencia.curitiba.pr.gov.br/>. Acesso em: 01 mar. 2018.

PRASS, F. S. Estudo Comparativo entre Algoritmos de Análise de Agrupamentos em Data Mining. 2004. 71 f. Dissertação (Mestrado em Ciência da Computação) Programa de Pós-Graduação em Ciência da Computação, Universidade Federal de Santa Catarina, Florianópolis. 